\title{
A Region Based Algorithm for Vessel Detection in Retinal Images
}

\author{
Ke Huang ${ }^{1}$ and Michelle Yan $^{2}$ \\ ${ }^{1}$ Department of Electrical and Computer Engineering, Michigan State University, \\ East Lansing, MI48824, USA* \\ ${ }^{2}$ Siemens Corporate Research, 755 College Road East, Princeton, NJ08540, USA
}

\begin{abstract}
Accurate retinal blood vessel detection offers a great opportunity to predict and detect the stages of various ocular and systemic diseases, such as glaucoma, hypertension and congestive heart failure, since the change in width of blood vessels in retina has been reported as an independent and significant prospective risk factor for such diseases. In large-population studies of disease control and prevention, there exists an overwhelming need for an automatic tool that can reliably and accurately identify and measure retinal vessel diameters. To address requirements in this clinical setting, a vessel detection algorithm is proposed to quantitatively measure the salient properties of retinal vessel and combine the measurements by Bayesian decision to generate a confidence value for each detected vessel segment. The salient properties of vessels provide an alternative approach for retinal vessel detection at a level higher than detection at the pixel level. Experiments show superior detection performance than currently published results using a publicly available data set. More importantly, the proposed algorithm provides the confidence measurement that can be used as an objective criterion to select reliable vessel segments for diameter measurement.
\end{abstract}

\section{Introduction}

The retinal vessels are the only part of the central circulation that can be optically observed in vivo. It offers an opportunity to non-invasively explore the relationship of systemic microvascular disease to cardiovascular disorders. For example, signs of retinopathy, e.g. microaneurysms, retinal hemorrhages and soft exudates, are the symptoms of microvascular damage from elevated blood pressure, diabetes, and other processes [1]. Changes in width of retinal vessels are the indication of both ocular diseases and cardiovascular disorders, such as glaucoma [2, hypertension [3], diabetic retinopathy [4] and congestive heart failure [5]. Retinal vessel diameters are often measured in a concentric zone surrounding the optic disc, yielding the value of arterio-venous ratio (AVR), an independent and significant risk factor measure used for examining many systemic diseases [6]. Lower AVR is commonly interpreted as an indicator of

\footnotetext{
* This work was completed when K. Huang was an intern at Siemens Corporate Research.
} 


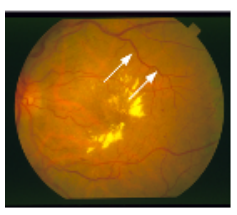

(a)

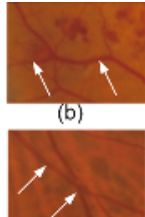

(c)

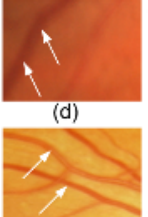

(e)

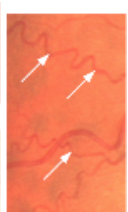

(f)

Fig. 1. Samples of an original retinal image (a) and different appearances of vessels. The large variation in appearance is caused by: (b) hemorrhage; (c) black background texture; (d) motion blurring; (e) different depths, diameters, contrast and central flex in the middle of arteries; (f) tortuosity. Arrows in the figure annotate the locations of some vessels.

arteriolar narrowing that is strongly associated with the onsets and effects of systemic diseases. In addition to diameter measurement, vessel detection is also used for retinal image registration [7, the optic disk localization [8], and as prior structural information in lesion detection [9].

Vessel detection in retinal images is a very challenging task due to several reasons. In the imaging process, retinal vessel structures at different depths are projected and overlapped onto the two-dimensional retinal image. A white strip called central reflex often stands out in the center of an artery and misleads the detection process. Uneven illumination, blurring, tortuosity and lesions of various appearances may also be present in the images. The width of vessel varies in a large range, from several pixels to tens of pixels. Fig. 11 illustrates an original retinal image with bright lesions and different appearances of retinal vessels. Physiological structures with blood are best contrasted in the green channel of color retinal images [10,11, 12, 13. Therefore, green channel image is used for vessel detection in the paper.

\subsection{Related Work}

Existing approaches for retinal vessel detection generally fall into two categories: tracking and classification. Thresholding can be viewed as classification with a simple classifier. An example of classification is introduced in [12], where the responses of an image to a set of matched filters are analyzed with threshold probing. In [13], ridge lines are first identified and more than 20 features are defined and computed for each pixel. A general process for tracking approaches starts with identifying some seed points, and the vessel structures recursively grow to their neighboring pixels from the seed points. In [14, a tracking algorithm following this paradigm is presented, where matched filters are used to analyze the neighboring pixels of currently detected vessels for recursive tracking. In [11], a tracking algorithm following similar steps is also proposed and verified. An algorithm combining thresholding and classification is proposed in [15], where the gray values of pixels are thresholded with decreasing values and results are refined by a classification process with a feature vector measuring properties such as angle, width, contrast and size. A combination of tracking 
and classification is introduced in [10, where vessel detection is implemented by combining the tracking algorithm introduced in [14] and classification with a 6dimensional feature vector that represents the likelihood of a pixel being inside a vessel.

\subsection{Our Proposed Method}

The blood vessel often appears to be a dark and continuous linear structure in retinal images. Normally, the boundaries of a short vessel segment are parallel to each other. To capture these visually salient properties, a region based Bayesian detection method is proposed for vessel detection in this paper, which works as follows. Cross sections of a vessel are first identified by searching boundary points along two parallel edges of an edge map. A confidence value is computed for each cross section to reflect its contrast to the background. The identified cross sections are then grouped into potential segments along the direction of a vessel. Each segment is associated with a confidence value based on the segment length and the variance of cross sections in width. Bayesian rule is introduced to combine the confidence values obtained at each step to generate a final confidence map, where a higher value represents a higher confidence of the segment being part of a vessel. By exploring the salient properties of retinal vessel at higher than the pixel level, contextual information can be effectively incorporated into the detection process. The confidence map in the proposed method offers a potential reference in the application where it needs to know how reliable of each detected vessel segment is. To the best of our knowledge, no current retinal vessel detection provides such a measurement.

The rest of this paper is organized as following. Section 2 discusses the implementation of region based Bayesian detection. Experimental results are presented in Section 3 and Section 4 concludes this paper.

\section{Region Based Bayesian Detection}

The proposed region based Bayesian detection is guided by first obtaining the quantitative measurements of the shape and continuity property, and then combining the measurements in the framework of Bayesian decision. Retinal vessels often appear to be darker in gray value than their neighbor regions, which implies that the intensity is a local property to be measured. Therefore, applying a single threshold to gray values of an entire image can not reliably separate vessel and non-vessel regions. To address this problem, shade correction [9] is used to compensate for the background variations across the whole retinal image. A conservative threshold is applied to the image obtained from the shade correction to generate a binary mask $B$, where the pixels with the value higher than the threshold are excluded from the following processing, as shown in Fig. 2 ,

\subsection{Shape Measurement}

An edge map is obtained by applying Canny edge detector to the green channel image. Since boundaries of a short vessel segment are normally parallel and close 


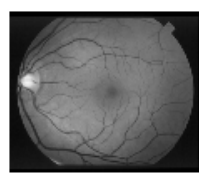

(a)

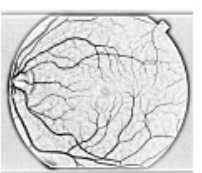

(b)

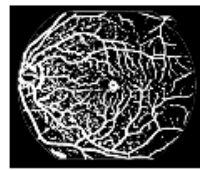

(c)

Fig. 2. Generating binary mask with shade correction: (a) green channel image; (b) image after shade correction; (c) binary mask $B$, where only the white regions are searched for potential vessels.

to each other, edges that are parallel to each other are first identified in the edge map. To achieve this goal, the edge map is searched horizontally and vertically. Taking searching row $r$ horizontally as an example: denote $P_{i}$ and $P_{j}$ as two neighbor edge points on row $r$, and $P_{i}$ and $P_{j}$ are considered as a pair of vessel boundary points only when both of the following conditions are satisfied:

1. $\left(P_{j}-P_{i}\right)>D_{l}$ or $\left(P_{j}-P_{i}\right)<D_{h}$

2. $\mu\left(r, P_{i}, P_{j}\right)<\mu\left(r, P_{i}-D_{t}, P_{i}-1\right)$ and $\mu\left(r, P_{i}, P_{j}\right)<\mu\left(r, P_{j}+1, P_{j}+D_{t}\right)$

The first item introduces the constrain on vessel width. The retinal image used in the experiment is with a size of $605 \times 700$. $D_{h}$ is set to 25 pixels and $D_{l}$ is set to 2 pixels to avoid missing any potential vessels. In the second item, the function $\mu\left(r, P_{i}, P_{j}\right)$ denotes the mean value of image intensity of the pixels between $P_{i}$ and $P_{j}$ in row $r$ of the corresponding green channel image. This constraint requires that the appearance of vessel pixels should be less or darker than its neighbor regions. $D_{t}$ is defined as the number of the neighbor pixels at each side and is set to 5 in the experiment. Once $P_{i}$ and $P_{j}$ are identified as a pair of vessel boundary points, a value $C 1$ is empirically defined to reflect the degree of the confidence associated with the cross section between $P_{i}$ and $P_{j}$ using the following formula:

$$
C 1=\left(E_{i}+E_{j}\right) e^{-\alpha_{1}\left|E_{i}-E_{j}\right|}
$$

where $E_{i}$ and $E_{j}$ are the edge intensity at the point $P_{i}$ and $P_{j}$, respectively. A larger $E_{i}$ or $E_{j}$ reflects a stronger image contrast between the vessel and its neighbors. However, because the two boundary points at a cross section should be close to each other in distance, a large difference in intensity between one boundary point to the other should be penalized. In the experiment, $\alpha_{1}$ is set to 0.05 for this penalization. All pixels along the cross section between $P_{i}$ and $P_{j}$ are assigned to the value $C 1$. The pixels not on any identified cross section are set to 0 . In this way, a $2 \mathrm{D}$ confidence map $S_{h}$ is obtained for shape measurement in the horizontal direction. The confidence map $S_{v}$ for vertical shape measurement can also be obtained in a similar way by searching the images vertically. Fig. B(a) and (b) demonstrate the shape confidence measurement in the horizontal and vertical directions, respectively.

The gradient information that is available in the edge detection process may be used for searching boundary points at cross sections. In the ideal case, the searching direction should follow the local gradient of each vessel boundary. How- 


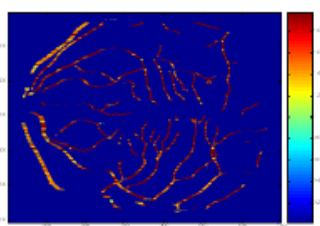

(a)

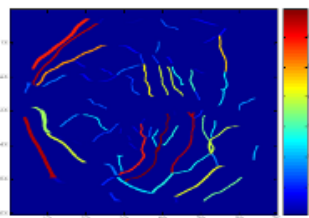

(c)

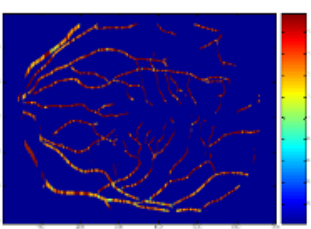

(b)

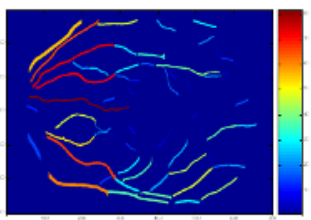

(d)

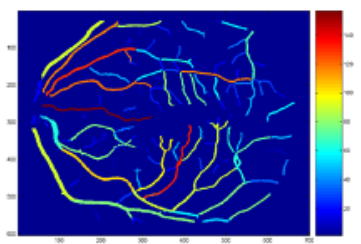

(e)

Fig. 3. Measured confidence maps of shape and continuity: (a) shape confidence map $S_{h}$ in the horizontal direction; (b) shape confidence map $S_{v}$ in the vertical direction; (c) continuity confidence map $C_{h}$ in the horizontal direction; (d) continuity confidence map $C_{v}$ in the vertical direction; (e) the final confidence map obtained using Bayesian rule. Red color represents high values and blue color represents low values.

ever, due to the noise and discrete representation of an image, not all boundary points can be uniquely paired and the searching lines may not cover all pixels inside each vessel segment. Therefore, we choose to horizontally and vertically search the image to make sure that every pixel is searched and a vessel segment at any orientation can be detected.

\subsection{Continuity Measurement}

In addition to the vessel structures, other non-vessel structures may also generate strong response in either or both of $S_{h}$ and $S_{v}$. One such example is the background region between two neighboring bright lesions, as shown in Fig. 4 . The salient property that could potentially separate the vessels from the rest is that the vessel often has an extended linear and continuous structure. This observation motivates us to further explore the contextual information by quantitatively measuring the continuity of the vessel.

The continuity is measured based on the cross sections that are identified in the section 2.1. The basic operation is to recursively connect each single cross section to form a continuous vessel segment, i.e., a vessel segment is detected in a similar fashion to the region growing, but using single cross section as the basic unit. Taking the cross sections identified along the horizontal direction as an example: an identified cross section $\left[P_{i}, P_{j}\right]$ at row $r$ is connected to another identified cross section $\left[P_{i}^{\prime}, P_{j}^{\prime}\right]$ at row $r+1$ only when both the following two conditions are satisfied:

1. The two cross sections overlap along the vertical direction.

2. The difference in width between the two cross sections must be less than $\lambda$, a predefined threshold, which is set to 5 in the experiment. 


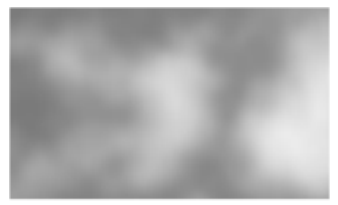

(a)

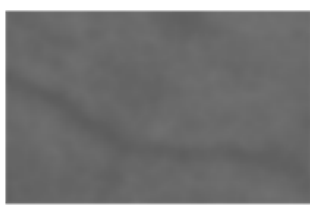

(b)

Fig. 4. Illustration of continuity: (a) the background region between the two bright lesions has two strong parallel, but short boundaries; (b) a less contrasted vessel that has an extended continuous linear structure.

In this way, the cross sections are connected to form potential candidates of vessel segment. Each potential vessel segment is assigned with a confidence value $C 2$ that is determined by the two factors: the total number of cross sections in the segment $(L)$ and the width variance of cross sections $(\sigma)$ in the segment, as empirically defined in the following formula:

$$
C 2=e^{-\alpha_{2} * \sigma} \ln (L)
$$

where $\alpha_{2}$ is a positive weighting factor, set to 0.3 in the experiment. Higher confidence values are assigned to vessel segments with a larger number of cross sections and a smaller width variance. After assigning all pixels on a vessel segment with their corresponding $C 2$ values and the pixels not on any vessel segment with the value 0 , a $2 \mathrm{D}$ confidence map $C_{h}$ is obtained to quantitatively measure the continuity of cross sections in the horizontal direction. Similarly, A 2 D confidence map $C_{v}$ can be obtained by connecting the cross sections identified vertically. An illustration of $C_{h}$ and $C_{v}$ is shown in Fig. 3 (c) and (d), where it is clear that this measurement effectively incorporates the contextual information for identifying vessels.

\subsection{Bayesian Decision and Post Processing}

There are four quantitative measurements $\left(S_{h}, S_{v}, C_{h}, C_{v}\right)$ associated with each potential vessel segment. Bayesian rule is introduced here to statistically combine the four measurements and provide one confidence map for each potential vessel segment. With the horizontal measurements $\left(S_{h}, C_{h}\right)$, the probability of being vessel given $S_{h}$ and $C_{h}$ can be computed with Bayesian rule as following:

$$
P\left(\text { Vessel } \mid S_{h}, C_{h}\right)=\frac{P(\text { Vessel }) P\left(S_{h}, C_{h} \mid \text { Vessel }\right)}{P\left(S_{h}, C_{h}\right)}
$$

Note that all pixels on a vessel segment have the same $C_{h}$ value, but may have different $S_{h}$ values. So the averaged $S_{h}$ of each vessel segment is used in the equation (3). To make the problem attackable, we assume $S_{h}$ and $C_{h}$ are independent of each other and $P(V e s s e l)$ is a uniform distribution. Therefore, the equation (3) is simplified as

$$
P\left(\text { Vessel } \mid S_{h}, C_{h}\right) \propto P\left(S_{h} \mid \text { Vessel }\right) P\left(C_{h} \mid \text { Vessel }\right)
$$


Thus, the confidence map $M_{h}$ is obtained by multiplying $S_{h}$ and $C_{h}$ element by element. Similarly, $M_{v}$, the confidence map for the vertical direction can also be obtained by multiplying the $2 \mathrm{D}$ map $S_{v}$ and $C_{v}$. We define the final confidence $\operatorname{map} M=\max \left(M_{h}, M_{v}\right)$. Fig. 3 (e) illustrates the final result obtained using this method.

Sudden changes in vessel width at bifurcations cause the vessel segment connecting process described in Section 2.2 to fail. To connect different vessel segments at bifurcations, a simple method for region filling is applied. First, all regions in binary mask $B$ are set to zero, where their corresponding values in $M$ are not zero. For the remaining small isolated regions in $B$, if one region can connect multiple regions that correspond non-zero values in $M$, the region is then used for region filling and the corresponding $M$ value is defined as the minimum in $M$. The non-maxima suppression introduced in [15] is recursively applied to the vessel boundary as a post processing step to reduce the boundary widening effect caused by Gaussian filtering in the Canny edge detection.

\section{Experimental Results}

The STARE data set 12 is used to evaluate the proposed algorithm and to facilitate performance comparison against several published papers using the same data set. Hand-labeled vessels as ground truth of 20 color retinal images are included in the data set. The images are with a size of $605 \times 700$ pixels in RGB format.

The performance of the proposed vessel detection method is measured using the Receiver Operating Characteristic (ROC) curve described in [12. ROC curves reported in [12] and [15] are duplicated in the paper for the purpose of comparison. A better result reported in 13 is not included, since the method is based on a high-dimensional feature vector with supervised learning that requires extensive training samples. The comparison of ROC curves of the proposed algorithm and those in [12, 15] is shown in Fig. 5. The result shows that the proposed algorithm achieves a better performance on the vessel detection with the STARE data set. Visual inspection indicates that the results with the false positive rate higher than 0.04 are too noisy to be useful in the applications like width measurement. Two examples of the vessel detection results, one with lesions and the other without lesions, are also shown in Fig. 5. The existence of lesions makes the vessel detection more difficult, as seen in the figure.

As discussed in Section 1.2, the accurate detection is one of the goals of the proposed algorithm. Since no vessel detection method is perfect, another goal that may be more important for vessel diameter measurement is to provide a measure for the detection reliability of each vessel segment. The confidence map in the proposed method provides such a measurement that may have potential to guide the vessel selection process. As one example shown in Fig. 3. (e), the vessel segments that are more reliably detected have higher values in the confidence map. 

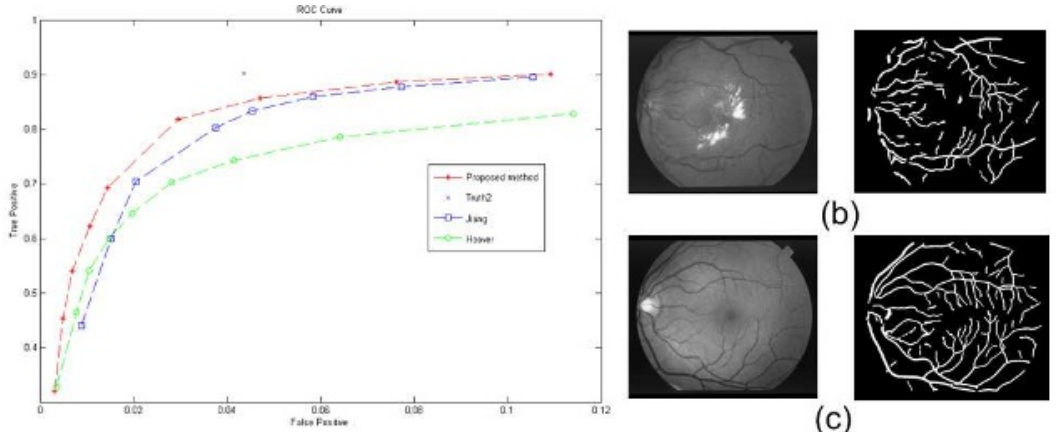

(b)

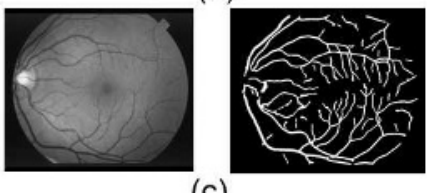

(a)

Fig. 5. Experimental results: (a) ROC curves with the STARE data set: curve with legend "proposed method" is obtained with the method proposed in this paper; curve with legend "Hoover" is duplicated from 12, curve with legend "Jiang" is duplicated from [15]; the cross point with legend "Truth2" is obtained with another manually labeled result of the STARE data, used as the performance bound for the computational methods [12,15]; (b) A result from a retinal image with bright lesions; (c) A result from a healthy retinal image.

\section{Summary and Future Research}

This paper explores the appearance and shape properties of vessel for retinal vessel detection, and a better detection accuracy is achieved. As a by-product, the proposed method provides a confidence value describing how reliable each detected vessel segment is detected.

There are other interesting topics of retinal vessel detection that are not discussed in this paper, but are ongoing research topics, for example, (1) sensitivity analysis of the parameters used; (2) extension of the method to the supervised learning case, where manually labelled vessel maps are available for training. In this setting, a Bayesian formula for computing likelihood can be obtained. (3) Experiments on larger data set of retinal images and a comprehensive evaluation of the method.

\section{References}

1. Wong, T., Klein, R., et al.: Retinal microvascular abnormalities and their relationship with hypertension, cardiovascular disease, and mortality. Survey of Ophthalmology 46(1) (2001) 59-80

2. Mitchell, P., Leung, H., et al.: Retinal vessel diameter and open-angle glaucoma: the blue mountains eye study. Ophthalmology 112(2) (2005) 245-250

3. Wong, T., Mitchell, P.: Hypertensive retinopathy. The New England Journal of Medicine 351(22) (2004) 2310-2317

4. Klein, R., Klein, B., et al.: The relation of retinal vessel caliber to the incidence and progression of diabetic retinopathy: Xix: the wisconsin epidemiologic study of diabetic retinopathy. Archives of Ophthalmology 122(1) (2004) 76-83 
5. Wong, T., Rosamond, W., et al.: Retinopathy and risk of congestive heart failure. Journal of the American Medical Association 293(1) (2005) 63-69

6. Hubbard, L., Brothers, R., et al.: Methods for evaluation of retinal microvascular abnormalities associated with hypertension/sclerosis in the atherosclerosis risk in communities study. Ophthalmology 106(12) (1999) 2269-2280

7. Shen, H., Stewart, C., et al.: Frame-rate spatial referencing based on invariant indexing and alignment with application to online retinal image registration. IEEE Trans. on Pattern Analysis and Machine Intelligence 25(3) (2003) 379-384

8. Foracchia, M., Grisan, E., et al.: Detection of optic disc in retinal images by means of a geometrical model of vessel structure. IEEE Trans. on Medical Imaging 23(10) (2004) 1189-1195

9. Huang, K., Yan, M.: A local adaptive algorithm for microaneurysms detection in digital fundus images. In: ICCV Workshop of Computer Vision for Biomedical Image Applications. (2005)

10. Sofka, M., Stewart, C.: Retinal vessel extraction using multiscale matched filters, confidence and edge measures. Technical report, Department of Computer Science, Rensselaer Polytechnic Institute, NY, USA (2005)

11. Wu, D., Zhang, M., et al.: On the adaptive detection of blood vessels in retinal images. Technical report, Department of Computer Science, Texas A\&M University, TX, USA (2005)

12. Hoover, A., Kouznetsova, V., et al.: Locating blood vessels in retinal images by piecewise threshold probing of a matched filter response. IEEE Trans. on Medical Imaging 19(3) (2000) 203-210

13. Stall, J., Abramoff, M., et al.: Ridge-based vessel segmentation in color images of the retina. IEEE Trans. on Medical Imaging 23(3) (2004) 501-509

14. Can, A., Shen, H., et al.: Rapid automated tracing and feature extraction from retinal fundus images using direct exploratory algorithms. IEEE Trans. on Information Technology in Biomedicine 3(2) (1999) 125-138

15. Jiang, X., Mojon, D.: Adaptive local thresholding by verification-based multithreshold probing with application to vessel detection in retinal images. IEEE Trans. on Pattern Analysis and Machine Intelligence 25(1) (2003) 131-137 